NASZA DERMATOLOGIA Online OUR DERMATOLOGY Online

Source of Support: Nil

Competing Interests: None

\section{EVALUATION OF THE EFFECT OF INJECTION OF DUTASTERIDE AS MESOTHERAPEUTIC TOOL IN TREATMENT OF ANDROGENETIC ALOPECIA IN MALES}

\author{
Nagat Sobhy, Hala Aly, Adel El Shafee, Marwa El Deeb \\ Department of Dermatology \& Venereology Faculty of medicine, Alexandria \\ University, Egypt
}

Corresponding author: Ass Prof. Nagat Sobhy Mohamad nana_dermatology@yahoo.com

\begin{abstract}
Introduction: Androgenetic alopecia (AGA) is hereditary and androgen dependent, progressive thinning of the scalp hair that follows a defined pattern.

Aim ot the work: is to evaluate the efficacy and safety of mesotherapy using dutasteride in treatment of androgenetic alopecia in males. Materials and Methods: Ninety male patients were randomly assigned into three groups; group A containing 30 patients who received pure dutasteride, group B of another 30 patients who received dutasteride containing solution and group C of the remaining 30 patients who received saline. Each group was given nine mesotherapy sessions. Assessment was done using trichogram, independent observer assessment of photographs and patients self assessment together with evaluation of possible systemic absorption using semenogram and serum dihydrotestosterone (DHT).

Result: Statistical analysis of the thrichogram results, the effect on semenogram and the serum level of dihydrotestosterone.showed that dutastride containing solution was the best.

Conclusion: Mesotherapy using dutasteride is a good option for treatment of male pattern hair loss; resulting in reduction or cessation of hair loss and promotion of new hair growth.
\end{abstract}

Key words: methotherapy; dutastride; male; androgenetic alopecia; trichogram

\section{Introduction}

Pattern hair loss (PHL), or androgenetic alopecia (AGA) or common baldness, is the most common cause of hair loss in men [1-3]. It is distinctive due to the pattern of progression of the scalp hair loss.

Dutasteride shares important characteristics with finasteride. While finasteride inhibits type II 5- $\alpha$ reductase, dutasteride inhibits both type I and II isoenzymes [4-6]. However, there is evidence that dutasteride is three times as potent as finasteride at inhibiting type II $5-\alpha$ reductase and more than 100 times as potent at inhibiting type I enzyme. This suggests enhanced efficacy over the existing finasteride, so scalp and serum levels of DHT are more affected i.e. dutasteride decreases serum DHT by more than 90\% [7], while finasteride decreases serum DHT by $70 \%[8,9]$.

The half-life of dutasteride is 4 weeks, compared with 6 to 8 hours for finasteride. This suggests that the effects on sexual function are longer lasting (several months) and more difficult to reverse [5]. Men being treated with dutasteride should not donate blood until they have been off of the medication for at least 6 months $[5,10]$.
- Side effects:

1) Sexual side effects including impotence (occurring in $69 / 817$ on finasteride $5 \mathrm{mg}$, vs. 55/813 on dutasteride), decreased libido (in $46 / 817$ on finasteride vs. 39/813 on dutasteride), ejaculation disorder (in 12/817 on finasteride vs. $10 / 813$ on dutasteride). There were no statistically significant differences between dutasteride-and finasteridetreated patients but there were statistically significant differences between dutasteride and placebo group initially [11]. These adverse events are characterized as either mild or moderate in severity and often resolve with continuation of the medication [12].

2) Effects on semen:

In a double-blind, placebo controlled trial of men given dutasteride $0.5 \mathrm{mg}$ daily or placebo; there were significant decrease from baseline in sperm count. Semen volume was decreased by a corresponding amount. There was also significant reduction in sperm motility. However, no significant changes were observed in sperm morphology [13]. 
Aim of the work:

The aim of the work is to evaluate the efficacy and safety of mesotherapy using dutasteride in treatment of androgenetic alopecia in males.

\section{Patients:}

- This study was carried out on 90 male patients complaining of pattern hair loss. They were recruited from the outpatient clinic of Dermatology, Andrology and Venereology Department, Alexandria Main University hospital.

\section{Inclusion criteria:}

- Age: 18-55 years.

- Free from clinically significant condition (Diabetes, history of stroke, history of any thromboembolic phenomenon or cancer).

- Not seeking pregnancy.

- Baseline semen analysis within normal range.

- Baseline serum DHT level within normal range

\section{Exclusion criteria:}

- Patients using the following medications: inhibitors of CYP3A4 (verapamil) or drugs with antiandrogen effect (finasteride) or hair growth promotors (minoxidil) in the last six months .

- Patients suffering from ejaculatory or erectile dysfunction.

\section{Methods}

Informed consent was taken from every patient and the study was approved by Alexandria university research ethics committee.

- Patients were randomly assigned into three groups:

- Group A: 30 patients who received mesotherapy injections of pure dutasteride. (Dutasteride $0.005 \%$ and polysorbate 80 in sodium chloride, Dallas, Texas).

- Group B: 30 patients who received mesotherapy injections of dutasteride containing solution. (Dutasteride $5 \mathrm{mg}$, dexapanthenol $500 \mathrm{mg}$, biotin $20 \mathrm{mg}$, pyridoxine $200 \mathrm{mg}$ per vial of $10 \mathrm{ml}$ i.e. $0.05 \%$ dutasteride, purchased from Mesodermal. USA).

- Group C: 30 patients who received mesotherapy injections of $0.9 \%$ saline.(control).

\section{All patients were subjected to the following.}

-1-Full history taking

-2-Clinical examination

- 3-Routine laboratory Investigations (CBC, FBS, LFT, RFT...)

- 4-Semen analysis before starting therapy and one week after last session

- 5-Serum DHT before starting therapy and one week after last session

- 6-Trichogram before starting therapy and one week after last session

- 7-Photographs of the scalp

Schedule:

- Once per week for four weeks.(week 0,1,2,3)

- Once every two weeks for one month.(week 5,7)

- Once per month for three months. (week 11,15,19)

\section{Results}

This study was carried out on 90 male patients complaining of pattern hair loss. Patients' age ranged from 18-55 years.

Duration of AGA ranged from 6 months up to 5 years. Family history was positive in $85 \%$ of cases.

Comparison between the effects of dutasteride, dutasteride containing solution and saline on trichogram: The results were more evident and good in group B:

By Wilcoxon Signed Rank test, there is statistically significant increase between anagen hair percent before treatment and after treatment $\left(\mathrm{Z}=-2.803, \mathrm{P}=0.005^{*}\right)$ but there is no statistically significant difference between catagen hair percent before treatment and after treatment. Also, there is statistically significant decrease between telogen hair percent before treatment and after treatment $\left(\mathrm{Z}=-2.803, \mathrm{P}=0.005^{*}\right)$. Regarding dystrophic hair percent, there is no statistically significant difference between before treatment and after treatment $(\mathrm{Z}=-0.561, \mathrm{P}=0.575)$. There is statistically significant increase between $\mathrm{A} / \mathrm{T}$ ratio before treatment [median $=1.5$, range (1-4)] and after treatment $(Z=-2.805$, $\left.\mathrm{P}=0.005^{*}\right)$. Finally, there is statistically significant increase between mean hair shaft diameter before treatment and after treatment $\left(\mathrm{Z}=-2.803, \mathrm{P}=0.005^{*}\right)$.

\section{Comparison between the effects of dutasteride, dutasteride} containing solution and saline on semenogram (one week after last session):

By Kruskal Wallis test, there is no statistically significant difference in semen volume, sperm concentration, sperm motility, sperm morphology between group A group Band group $\mathrm{C}\left(\mathrm{X}_{\text {KRUSKal Wallis }}^{2}=0.366, \mathrm{P}=0.833\right)$.

Serum dihydrotestosterone (DHT) (normal range: from 250 up to $990 \mathrm{pg} / \mathrm{ml}$ ).

Comparison between the effects of dutasteride, dutasteride containing solution and saline on DHT (one week after last session):

There was unexpected variation in results of serum DHT in the form of increase in serum DHT in group A while a decline in serum DHT in group B and group C could be explained by the wide normal range for serum DHT and the diurnal variations.

Patient self assessment (Tabl I).

By Monte Carlo significance test, there is no statistically significant difference in patient self assessment in the three groups (Monte Carlo $\mathrm{P}=0.089$ ).

Independent observer assessment (Tabl II) (Fig. 1, 2)

By Monte Carlo significance test, there is statistically significant difference between the three groups. It is revealed that in group A, the majority (70\%) perceived as no change (score $=0$ ), $20 \%$ as mild improvement (score $=1$ ), $10 \%$ as moderate improvement ( (core $=2)$, while group $B$ the majority were perceived as improved mildly or moderately $(40 \%$ had score $=1,40 \%$ had score $=2)$ and $20 \%$ perceived as no change (score $=0$ ) but neither groups were perceived to have been worse (score $=-1$ ). Finally, in group C 30\% was perceived as worsened, $40 \%$ as no change and $30 \%$ as mild improvement. $\left(\mathrm{P}=.026^{*}\right)$. These results had a similar trend to the results of trichogram being some improvement in groups $\mathrm{A}$ and $\mathrm{B}$, more in $\mathrm{B}$, as compared to control group. 


\begin{tabular}{|c|c|c|c|c|c|c|c|}
\hline & \multicolumn{2}{|c|}{ Dutasterise (group A) } & \multicolumn{2}{|c|}{$\begin{array}{l}\text { Dutasteride containing } \\
\text { solution (group B) }\end{array}$} & \multicolumn{2}{|c|}{ Saline (group C) } & \multirow[t]{2}{*}{ Sig. } \\
\hline & No. & $\%$ & No. & $\%$ & No. & $\%$ & \\
\hline Not satisfied & 12 & 40 & 3 & 10 & 18 & 60 & \multirow[t]{2}{*}{0.089} \\
\hline Satisfied & 18 & 60 & 27 & 90 & 12 & 40 & \\
\hline
\end{tabular}

Table I. Patient self assessment

* Significant level $<0.05$

\begin{tabular}{|c|c|c|c|c|c|c|c|}
\hline & \multicolumn{2}{|c|}{ Dutasterise (group A) } & \multicolumn{2}{|c|}{$\begin{array}{l}\text { Dutasteride containing } \\
\text { solution (group B) }\end{array}$} & \multicolumn{2}{|c|}{ Saline (group C) } & \multirow[t]{2}{*}{ Sig. } \\
\hline & No. & $\%$ & No. & $\%$ & No. & $\%$ & \\
\hline-1 & 0 & 0 & 0 & 0 & 9 & 30 & \multirow[t]{4}{*}{$0.026^{*}$} \\
\hline 0 & 21 & 70 & 6 & 20 & 12 & 40 & \\
\hline 1 & 6 & 20 & 12 & 40 & 9 & 30 & \\
\hline 2 & 3 & 10 & 12 & 40 & 0 & 0 & \\
\hline
\end{tabular}

Table II. Independent observer assessment

* Significant level $<0.05$

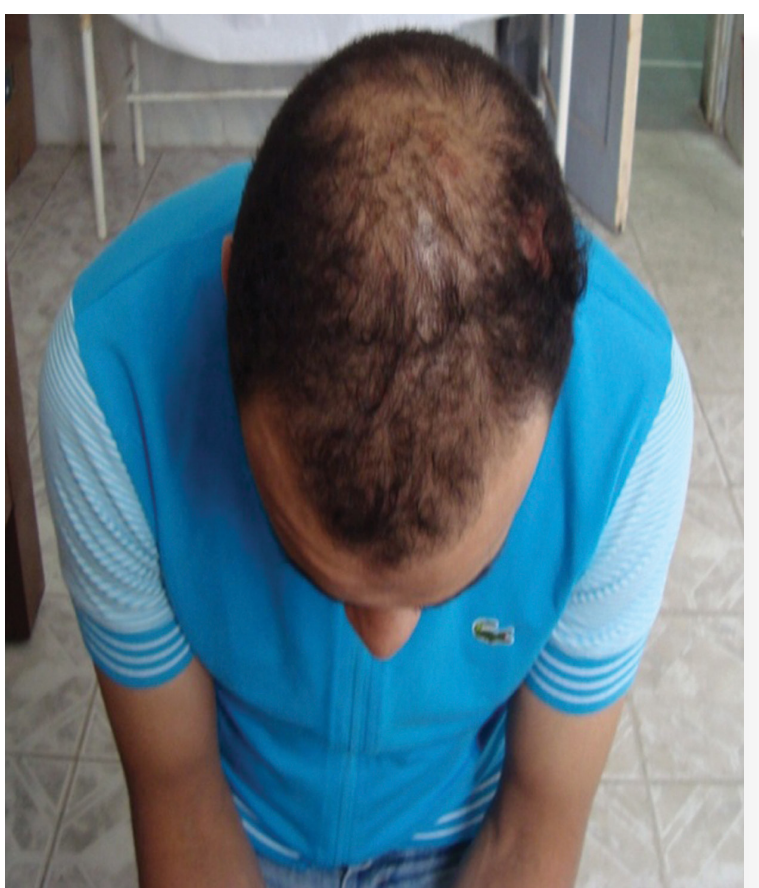

Figure 1. Group A. Before starting treatment (week 0).

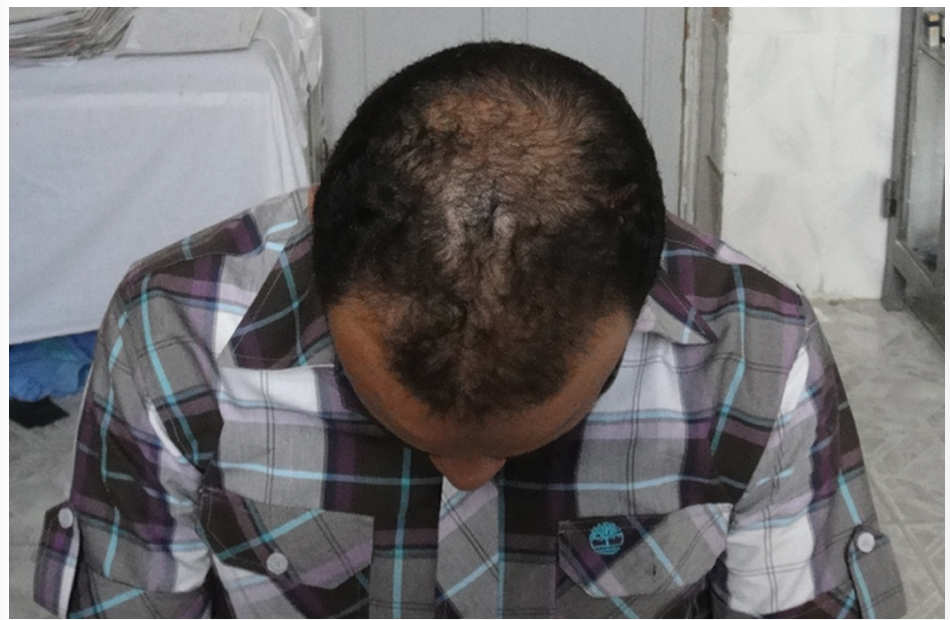

Figure 2. After receiving nine sessions (week 20)

\section{Discussion}

Androgenetic alopecia (AGA) or pattern hair loss (PHL) is the most common type of hair loss in men [1-3].

Options for management in men include doing nothing and accepting the cosmetic outcome, pharmacotherapy, hair transplantation, and cosmetic aids. Hair loss is progressive and does not improve or reverse without treatment [14]. Systemic $5 \alpha$-reductase inhibitors e.g. finasteride and dutasteride arrest progression of androgenetic alopecia in over $90 \%$ of men and partially reverse it in over $65 \%$ [3]
Few studies have been conducted on dutasteride for the treatment of MPHL (male pattern hair loss) [12]. Hesitancy about the widespread use of dutasteride in the treatment of MPHL results from its potential side effects on erectile, ejaculatory functions (several months) and fertility together with its long half life (4 weeks) [5]. The possibility of using dutasteride locally would minimize these systemic side effects [15].

The control group was included to establish the hypothesized role of trauma and the psychic element of therapy. 
Demographic data of the present study revealed that patients were around the same age (median for gp $A=29.5$ years, gp $\mathrm{B}=30$ years, gp $\mathrm{C}=28.5$ years), duration of alopecia (median for gp $\mathrm{A}=1.5$ year, gp $\mathrm{B}=4$ years, gp $\mathrm{C}=4$ years) and grade of alopecia, also, there was no significant difference regarding family history. There was no correlation between age, duration or grade of alopecia and the degree of improvement of hair loss. However, it was noted that positive family history was associated with better improvement of alopecia (anagen hair percent and mean hair shaft diameter), though correlation was not statistically significant.

In Abdallah $\mathrm{M}$ et al. [15], which is to the best of our knowledge, the only study conducted evaluating mesotherapy injection of dutasteride in treatment of MPHL, there was no significant difference between the two studied groups with respect to age, stage and duration of baldness. Meanwhile, there was no correlation between age of patients and stage of baldness with the scored improvement but there was a reversed correlation between duration of baldness with the scored improvement.

In the present study, analysis of results of trichogram revealed statistically significant increase in anagen hair percent in group B patients. While in group A; the increase was statistically insignificant. Regarding the control group $\mathrm{C}$; there was statistically insignificant decrease in anagen hair percent.

Meanwhile, there was statistically significant decrease in telogen hair percent in group B patients. While group A; showed statistically insignificant decrease. Group C; there was statistically significant increase in telogen hair percent. Reduction in telogen hair count by treatment is expected in responsive cases due to the resulting prolongation of anagen phase so fewer hairs are in telogen phase rather than direct effect of therapy since its duration is not altered in MPHL [16].

Concerning anagen/telogen (A/T) ratio, there was statistically significant increase in group B. While in group A; the increase was mild and not statistically significant. Regarding group $\mathrm{C}$, there was an expected statistically significant decrease in $\mathrm{A} / \mathrm{T}$ ratio.

In the present study, group A and B showed statistically significant increase in mean hair shaft diameter. While group $\mathrm{C}$ showed mild statistically insignificant increase in mean hair shaft diameter, this goes with the alleged role of trauma of mesotherapy injection in improvement of AGA. This was also noted in the insignificant decrease in anagen hair percent in the group $\mathrm{C}$, which possibly means that trauma slowed down the progression of hair loss.

Theoretically dutasteride would be expected to be more effective than finasteride for treating patients with MPHL [12]. This was detected in a randomized placebo controlled study in which the efficacy and safety of oral dutasteride at different doses $(0.05,0.1,0.5,2.5 \mathrm{mg})$ versus oral finasteride $(5 \mathrm{mg})$ in the treatment of MPHL was investigated by Oslen et al. [12].Patients were evaluated at 12 and 24 weeks. At 24 weeks, dutasteride 0.1 or $0.5 \mathrm{mg}$ daily were comparable to finasteride $5 \mathrm{mg}$ daily and dutasteride $2.5 \mathrm{mg}$ was superior to finasteride in stimulating hair growth and suppressing scalp and serum DHT.

From the present results, it could be presumed that improvement was partly due to modification of hair cycle dynamics and partly due to increasing the diameter of the existing hair.

Based on the results of trichogram coming in favour of dutasteride containing solution, it might be suggested that hair growth promoters in the preparation are having a synergistic role with dutasteride or the difference in concentration of the active ingredient being higher in dutasteride containg solution.

Stough D. [14] compared the efficacy of dutasteride $(0.5 \mathrm{mg} /$ day) vs. placebo in the treatment of male pattern hair loss in 17 pairs of identical twin males with androgenetic alopecia over 1 year period and found that dutasteride significantly reduces hair loss progression in men with male pattern hair loss.

Eun HC et al. [18] conducted a study on 153 men who were randomized to receive $0.5 \mathrm{mg}$ of dutasteride or placebo daily for 6 months. Mean change of hair counts from baseline to 6 months after treatment start was an increase of $12.2 / \mathrm{cm}^{2}$ in dutasteride group and $4.7 / \mathrm{cm}^{2}$ in placebo group and this difference was statistically significant. The improvement noticed in the placebo group was explained by a seasonal factor.

Previous studies confirmed the efficacy of oral dutasteride in treatment of MPHL [14,18] that was also detected using mesotherapy injection of dutasteride. The debate here is it a comparable efficacy or lower efficacy of mesotherapy vs. oral route that could be accepted if poses less incidence of side effects. One must mention that in our study, inspite of the statistically significant improvement noted in the trichogram of patients in the actively treated groups (A and B), this improvement was not so evident clinically.

Concerning patients self assessment there was no statistically significant difference between the three groups regarding satisfaction due to cessation of hair loss and the noticed improvement (60\% in group A, $90 \%$ in group B and $40 \%$ in group $\mathrm{C}$ were satisfied) and, moreover, their satisfaction could not be correlated to trichogram results. Even, the majority of patients including those who were not satisfied asked for more sessions which explain the psychological aspect of therapy. In contrast, Abdallah $\mathrm{M}$ et al. [15] revealed the active group was significantly more satisfied with treatment than the placebo group.

Independent observer assessment revealed statistically significant difference favoring the actively treated groups than the control group that was scored as mild-moderate improvement.

In an attempt to evaluate the possibility of systemic absorption and the resulting side effects, changes in semenogram were statistically insignificant but it must be mentioned that there was a decline in semen volume in group A, a decline in sperm concentration in group B, and a decline in sperm motility in group A and B. Meanwhile, sperm morphology showed no statistically significant difference between the three groups. When looking at these results, one can notice that a degree of systemic absorption took place and did affect spermatogenesis although the results were insignificant but there is a trend of decline in actively treated groups compared to the control group concerning semen volume, sperm concentration and motility. 
Although the decline was in the normal range, two cases ( 27 and 40 years old) in group B showed a decline in sperm concentration from 29 million $/ \mathrm{ml}$ to 7 million $/ \mathrm{ml}$ and a corresponding decline in sperm motility from $\mathrm{A}+\mathrm{B}=65 \%$ to $28 \%$ and a concentration from 30 million/ml to 10 million/ $\mathrm{ml}$ and motility from $60 \%$ to $40 \%$, respectively. By repeating the semenogram 3 months later values recovered to almost the pretreatment baseline.

Such serious data detected in the present study, should be investigated on a larger scale and in a dose-dependent manner but until establishment of these data certain precautions should be taken regarding regimen adjustment.

Moreover, dermatologists should be aware of the total dose injected during mesotherapy. In the present study, patients received 1.5-2 $\mathrm{ml}$ per session (equivalent to $0.075-0.1 \mathrm{mg}$ for group A and 0.75-1 mg for group B) for 9 sessions i.e. a total of $13.5-18 \mathrm{ml}$ of mesotharpy injections, given that the concentration of dutasteride in pure solution ( $\mathrm{gp} \mathrm{A}$ ) is $0.05 \mathrm{mg} / \mathrm{ml}$ and in dutastreide containing solution (gp B) is $0.5 \mathrm{mg} / \mathrm{ml}$, then the cumulative dose of dutatseride for gp $\mathrm{A}$ is $0.675-0.9 \mathrm{mg}$ and for gp B is $6.75-9 \mathrm{mg}$. Taking in consideration the long half life of dutasteride [5] and the possible effect on spermatogenesis.

When compared with systemic dutasteride therapy, results were similar to a study conducted with Amory JK et al. [13] on 99 healthy men randomly assigned to receive 0.5 $\mathrm{mg}$ dutasteride or $5 \mathrm{mg}$ finasteride or placebo once daily for one year and evaluated for semen parameters and serum hormones (DHT and T). The study revealed statistically significant decline from baseline in total sperm count, semen volume, and sperm motility but not sperm morphology in both treatment groups as compared to the placebo that were recovered 24 weeks after drug discontinuation. Also, $5 \mathrm{AR}$ inhibitors significantly suppress serum DHT and transiently increase serum Testosterone. That study also declared the need for further investigations to establish the exact site and mechanism of action underlying the effect of 5AR inhibition on spermatogenesis.

Oslen etal. [12] found that scalp and serum dihydrotestosterone levels significantly decreased, and testosterone levels significantly increased, in a dose-dependent fashion with oral dutasteride tharpy, which returned to baseline levels 12 weeks after termination of therapy for $0.05 \mathrm{mg}$ and $0.1 \mathrm{mg}$ groups.

The question elaborated whether the extent of systemic absorption following mesotherpay is equal to or less than following oral administration of dutasteride given that the scalp is highly vascular.

In our study, results of serum DHT were contradictory and not reliable since there were statistically significant increase in group A but statistically insignificant decline in gp B and $\mathrm{C}$. This could be attributed to the wide normal range of DHT $(250-990 \mathrm{pg} / \mathrm{ml})$ and its diurnal variation, therefore it is better to be assessed together with serum T and scalp DHT. However, it is unknown to what extent DHT contributes to spermatogenesis. Men with $5 \alpha$ reductase type 2 deficiency have markedly impaired spergmatogenesis but they also have undescended testes which could contribute to the defect in sperm production. [17].

In addition, none of our patients complained of decreased libido, erectile or ejaculatory dysfunction as well as in the previously mentioned mesotherapy study [15]. When compared with oral dutaseride used in a study carried by Andriole GL and Kirby R [11] there was a modestly elevated incidence of impotence, decreased libido, ejaculation disorders, and gynaecomastia in the group who received dutasteride $0.5 \mathrm{mg}$ /day and that was statistically significant as compared to placebo. But Eun HE et al. [18] revealed in a recent study that no significant difference was observed between the two studied groups (group received $0.5 \mathrm{mg} / \mathrm{d}$ dutasteride and group received placebo) in terms of sexual function. A question to be raised in the context of such findings whether sexual function can be affected by oral dutasteride or systemic absorption of dutasteride during mesotherapy.

\section{Conclusion}

1. Mesotherapy using dutasteride is a good option for treatment of MPHL resulting in reduction or cessation of hair loss and promotion of new hair growth.

2. The proposed mechanism of action of dutasteride is partly due to modification of hair cycle dynamics and partly through increasing hair shaft diameter of the existing hairs.

3. The role of trauma of injection in the outcome of mesotherapy is mild.

4. The psychological impact of mesotherapy on the patients undertaking it is considerable and remarkably guides their satisfaction.

5. Systemic absorption of dutasteride following mesotherapy with its deleterious effect on spermatogenesis is possible especially with unlimited injections.

6. Effect on sexual function by dutasteride mesotherapy is questionable.

7. Mesotherapy using dutasteride is not recommended in patients seeking pregnancy, patients with borderline or abnormal semenogram and patients with ejaculatory or erectile dysfunctuion.

8. Further research to establish the exact changes in hair quality and quantity by dutasteride as mesotherapy on larger number of patients.

\section{REFERENCES}

1. Tully AS, Schwartzenberger J, Studdiford J: Androgenic Alopecia Review Article. J Men Health. 2010;7:270-7.

2. Sinclair RD: Androgenetic alopecia as an early marker of benign prostatic hyperplasia. J Am Acad Dermatol. 2012;66:401-8.

3. Sinclair RD: Male androgenetic alopecia. JMHG. 2004;1:31927.

4. Dutasteride.com [ online] 2005 [cited 2010 Aug 4]. Available from: http://www.dutasteride.com/dutasteride-chemistry.html

5. Rogers NE, Avram MR: Medical treatments for male and female pattern hair loss. J Am Acad Dermatol. 2008;59:54766; quiz 67-8.

6. Bramson HN, Hermann D, Batchelor KW, et al: Unique preclinical characteristics of GG745, a potent dual inhibitor of 5-AR. J Pharmacol Exp Ther. 1997;282:1496-502.

7. Clark RV, Hermann DJ, Cunningham GR, Wilson TH, Morrill BB, Hobbs S: Marked suppression of dihydrotestosterone in men with benign prostatic hyperplasia by dutasteride, a dual 5alpha-reductase inhibitor. J Clin Endocrinol Metab. 2004;89:2179-84. 
8. Dallob AL, Sadick NS, Unger W, Lipert S, Geissler LA, Gregoire SL, et al: The effect of finasteride, a 5-alpha-reductase inhibitor, on scalp skin testosterone and dihydrotestosterone concentrations in patients with male pattern baldness. J Clin Endocrinol Metabol. 1994;79:703-6.

9. Avodart clinical pharmacology [online] 2010 [cited 2010 Jan 20] Available from: http://www.rxlist.com/avodart-drug.htm.

10. Avodart warnings and precautions [online] 2010 [cited $2010 \mathrm{Jan}$ 20]. Available from: http://www.rxlist.com/avodart-drug.htm.

11. Andriole GL, Kirby R: Safety and tolerability of the dual 5alpha-reductase inhibitor dutasteride in the treatment of benign prostatic hyperplasia. Eur Urol. 2003;44:82-8.

12. Olsen EA, Hordinsky M, Whiting D, Stough D, Hobbs S, Ellis ML, et al: The importance of dual 5alpha-reductase inhibition in the treatment of male pattern hair loss: results of a randomized placebo-controlled study of dutasteride versus finasteride. J Am Acad Dermatol. 2006;55:1014-23.

13. Amory JK, Wang C, Swerdloff RS, Anawalt BD, Matsumoto AM, Bremner WJ, et al: The effect of 5 alpha reductase inhibition with dutasteride and finasteride on semen parameters and serum hormones in healthy men. J Clin Endocrinol Metab. 2007;92:165965.
14. Stough D: Dutasteride improves male pattern hair loss in a randomized study in identical twins. J CosmetDermatol.2007;6:9-13. 15. Abdallah M, El-Zawahry K, Besar H: Mesotherapy using dutastreide-containing solution in male pattern hair loss: a controlled pilot study. PALD. 2009;20:137-45.

16. Van Neste D, Fuh V, Sanchez-Pedreno P, Lopez-Bran E, Wolff $\mathrm{H}$, Whiting D, et al: Finasteride increases anagen hair in men with androgenetic alopecia. Br J Dermatol. 2000;143:804-10.

17. Andriole G, Bruchovsky N, Chung LW, Matsumoto AM, Rittmaster R, Roehrborn C, et al: Dihydrotestosterone and the prostate: the scientific rationale for 5 alpha reductase inhibitors in the treatment of benign prostatic hyperplasia. J Urol. 2004;172:1399403.

18. Eun HC, Kwon OS, Yeon JH, Shin HS, Kim BY, Ro BI, et al: Efficacy, safety, and tolerability of dutasteride $0.5 \mathrm{mg}$ once daily in male patients with male pattern hair loss: a randomized, doubleblind, placebo-controlled, phase III study. J Am Acad Dermatol. 2010;63:252-8. 